\title{
Continuous cardiovascular hemodynamics monitoring with pressure recording analytical method in patients under spinal anesthesia for elective cesarean section: a pilot study
}

\author{
Xiaoyu Zhang ${ }^{1,2 \#}$, Tao Xu ${ }^{1,2 \#}$, Lijie Jia ${ }^{1,2}$, Huimin Cao ${ }^{1,2}$, Zifeng $\mathrm{Xu}^{1,2}$ \\ ${ }^{1}$ Department of Anesthesiology, International Peace Maternity \& Child Health Hospital, Shanghai Jiao Tong University, School of Medicine, \\ Shanghai, China; ${ }^{2}$ Shanghai Key Laboratory of Embryo Original Diseases, Shanghai, China \\ Contributions: (I) Conception and design: X Zhang, Z Xu; (II) Administrative support: Z Xu; (III) Provision of study materials or patients: All authors; \\ (IV) Collection and assembly of data: All authors; (V) Data analysis and interpretation: All authors; (VI) Manuscript writing: All authors; (VII) Final \\ approval of manuscript: All authors. \\ \#These authors contributed equally to this work. \\ Correspondence to: Zifeng Xu. Department of Anesthesiology, International Peace Maternity \& Child Health Hospital, Shanghai Jiao Tong University, \\ School of Medicine, Shanghai 200080, China. Email: xuzf@shsmu.edu.cn.
}

Background Pressure recording analytical method (PRAM) of MOSTCARE is a minimally invasive
system based on mathematical analysis of arterial pressure profile changes and allows continuous recording
of hemodynamic measurements. Herein, we aimed to use the MostCare system to investigate the
hemodynamics of patients receiving spinal anesthesia during elective cesarean section. Methods: In this observational study, we recruited 17 patients scheduled for elective cesarean section. For each patient, we acquired continuous recordings of hemodynamic parameters at 14 key timepoints: surgical admission (two baseline parameters), immediately after the administration of spinal anesthesia, six subsequent time points $(2,4,6,8,10$, and $12 \mathrm{~min}$ after anesthesia), during peritoneum and uterine incision, during delivery, following the administration of oxytocin, and after surgery had been completed. Statistical analysis was carried out by repeated measures analysis of variance (ANOVA).

Results: During a twelve min period after spinal anesthesia, we observed significant reductions in mean arterial pressure (MAP), dicrotic pressure (Pdic), cardiac index (CI), stroke volume index (SVI), $\mathrm{dP}_{\mathrm{dt}} \mathrm{t}_{\max }$, and cardiac cycle efficiency (CCE) $(\mathrm{P}<0.05$ for all). However, systemic vascular resistance index (SVRI) was reversely correlated to $\mathrm{CI}$ and increased significantly after spinal anesthesia $(\mathrm{P}<0.05)$. These hemodynamic parameters return to near basal values after peritoneum incision. Furthermore, there were significant fluctuations in MAP, Pdic and CI after oxytocin administration $(\mathrm{P}<0.05)$.

Conclusions: PRAM of MostCare system revealed significant changes in key hemodynamic parameters undergoing cesarean section with spinal anesthesia. It enables clinicians gain a much better understanding of hemodynamics of parturients and optimize clinical management strategies.

Trial registration: This study was registered at http://www.chictr.org.cn on 16, July, 2019. No. ChiCTR1900024566.

Keywords: Cesarean; spinal anesthesia; pressure recording analytical method (PRAM); hemodynamics monitoring

Submitted Mar 16, 2021. Accepted for publication May 21, 2021.

doi: 10.21037/apm-21-598

View this article at: http://dx.doi.org/10.21037/apm-21-598 


\section{Introduction}

Cesarean section is the most commonly performed surgical procedure in the world; over 1.5 million sections are carried out in the USA each year (1). Spinal anesthesia is the most widespread technique for elective cesarean sections; this is because this technique is simple to carry out, has a low failure rate, and works quickly and effectively. However, severe hemodynamic disturbances occur commonly during spinal anesthesia; these disturbances may be associated with a number of adverse events relating to the neonate and mother $(2,3)$. The current technique used to monitor hemodynamics in such situations is the intermittent measurement of blood pressure. This is a non-invasive method and typically involves the measurement of blood pressure every minute, or even less frequently, without assessing oxygen delivery or cardiac output (CO) (4). Consequently, we have a very poor understanding of the actual effect of spinal anesthesia during cesarean section upon critical hemodynamic responses, including systemic vascular resistance (SVR) and changes in CO. It is vital that we monitor $\mathrm{CO}$ and $\mathrm{O}_{2}$ continuously during delivery as these parameters represent a direct reflection of oxygenation status of both the pregnant patients and fetus.

Continuous hemodynamic monitoring of arterial pressure, together with $\mathrm{CO}$, permits clinicians to calculate SVR and provides anesthetists with real-time data that will help clinicians to determine whether a hypotensive state is predominantly due to vasodilatation, a pump issue, or a reduction in blood volume (5). Recent advances in technology and science have led to the development of a new minimally-invasive system for analyzing pulse contours over extended periods of time. MostCare (Vytech Health srl, Padova, Italy) is a minimally invasive system based on pressure recording analytical method (PRAM). This system is minimally-invasive, permits hemodynamic monitoring, and only requires cannulation of the radial artery. Several publications have validated the PRAM system, and have also compared the PRAM system with pulmonary artery catheterization (PAC) (6,7). The PRAM system uses a mathematical algorithm to analyze changes in the profile of arterial blood pressure and permits the continuous recording of heart rate (HR), SVR, blood pressure, stroke volume (SV), cardiac index (CI), CO, cardiac cycle efficiency $(\mathrm{CCE})$, and $\mathrm{dP} / \mathrm{dt}_{\max }(8,9)$. Consequently, the PRAM system may help us to improve our knowledge of perioperative hemodynamics during obstetric anesthesia and allow us to create appropriate strategies for the clinical management of patients undergoing spinal anesthesia.

Considering the rigorous control of spinal anesthesiarelated hemodynamic responses in parturients and the recent advancement in minimally invasive hemodynamic monitoring, we aimed to use the MostCare system to investigate the hemodynamics of patients receiving spinal anesthesia during elective cesarean section in this observational study. We also dicuss the potential benefits of monitoring hemodynamic parameters in real time in the context of patient management. We present the following article in accordance with the MDAR reporting checklist (available at http://dx.doi.org/10.21037/apm-21-598).

\section{Methods}

\section{General information}

This observational study was approved by the Ethics Committee of International Peace Maternity \& Child Health Hospital (GKLW2015-65). The study was conducted in accordance with the Declaration of Helsinki (as revised in 2013). All of the patients involved in our study provided written and informed consent. In total, we recruited 20 pregnant women. All of these women had a stage 1 physical status according to the American Society of Anesthesiologists (ASA I) and were scheduled for elective cesarean section using spinal-epidural anesthesia between July and August 2019. Women were excluded if they had contraindications to regional anesthesia, were below the age of 18 or above the age of 40 , and if the gestational age was less than 36 weeks or more than 41 weeks. Women were also excluded if they were morbidly obese [body mass index (BMI) $\geq 40$ ], hypertensive, emergency cases, pre-eclamptic, or suffering from cerebrovascular or cardiovascular disease, diabetes mellitus, placenta previa, if they were suffering from a known anxiety disorder, autonomic neuropathy, intrauterine growth retardation, or were taking any form of medication. This study was registered at http://www.chictr. org.cn on 16, July, 2019. No. ChiCTR1900024566.

\section{Anesthesia procedure and monitoring}

None of the recruited women received premedication or prophylactic antiemetics before spinal anesthesia. Prior to induction, all women were fasted for at least 2 hours (clear liquid) and 6 hours (solid flood). An 18-gauge (G) cannula was inserted into the vein on the right hand. We then maintained the patency of the cannula by titrating 
Ringer's solution $(10 \mathrm{~mL} / \mathrm{kg} / \mathrm{h})$ immediately prior to the initiation of spinal anesthesia; this was maintained until surgery was complete. The temperature of operating room was maintained at $22{ }^{\circ} \mathrm{C}$ and all the pregnant women were transferred from the ward to the operating room without any additional warming. Once in the operating room, all women were fitted with a routine non-invasive monitor (IntelliVue MP30, PHILIPS Healthcare, Boblingen, Germany), including electrocardiography and pulse oximetry. The MOSTCARE monitor was connected via a $16-\mathrm{G}$ catheter that was inserted into the left radial artery. Patient data (body weight and height) were recorded in the MOSTCARE system. Then, we checked that the arterial line waveform was good quality. Next, we zeroed the system and began collecting hemodynamic data; the data was continuously recorded throughout the perioperative period.

A primary concern about PRAM reliability is related to the quality of the recorded arterial pressure signal. Patientrelated causes of inappropriate signal acquisition may be due to the hemodynamic instability or arrhythmias, such as severe hypotension and tachycardia. In order to improve the reliability of PRAM system, we maintain the parturients hemodynamics stability by $15^{\circ}$ left lateral tilt, controlling sensory block level and vasopressors. Parturients with severe post-spinal hypotension were excluded from the study.

For standard combined spinal-epidural anesthesia, each woman was placed in the full right-lateral decubitus position with head down and knees bent. Combined spinal-epidural anesthesia was administered into the L3-4 interspace using the needle-through-needle method. First, we inserted a 16-G Tuohy needle into the epidural space by confirming that there was a loss of resistance to $1 \mathrm{~mL}$ of air. Then we introduced a 26-G pencil-point spinal needle through the Tuohy needle and into the dura mater. After we confirmed the presence of free-flowing clear cerebrospinal fluid (CSF) from the spinal needle, a solution of $0.5 \%$ isobaric ropivacaine was prepared by diluting $2 \mathrm{~mL}$ of $0.75 \%$ ropivacaine with CSF to a final volume of $3 \mathrm{~mL}$. Next, we injected approximately $2.4 \mathrm{~mL}$ of ropivacaine solution into the subarachnoid space at a rate of $0.15 \mathrm{~mL} / \mathrm{sec}$. The epidural catheter was carefully inserted into the epidural space by 3.5 to $4.0 \mathrm{~cm}$, and was then secured. Patients were immediately returned to the supine position with a $15^{\circ} \mathrm{left}$ lateral tilt until the infant was delivered. A nasal cannula was used to supply the patient with oxygen (at a rate of $4 \mathrm{~L} / \mathrm{min}$ ). We evaluated the level of sensory blockade by testing the loss-of-cold sensation. We carried out this test 2 min after spinal injection and used $70 \%$ alcohol swabs to provide cold stimuli. If the level of the sensory was below T6, we injected further $4-10 \mathrm{~mL}$ boluses of $2 \%$ lidocaine via the epidural catheter until we achieved a sensory block that extended to the T6 level. These patients were excluded from further analysis.

\section{The study protocol}

All of the hemodynamic parameters were collected by a blinded observer who was not involved in anesthesia or patient management. A range of hemodynamic measurements were recorded continuously throughout the operation, including HR, mean arterial pressures (MAP, $\mathrm{mmHg}$ ), dicrotic pressure (Pdic, $\mathrm{mmHg}$ ), stroke volume index $\left(\mathrm{SVI}, \mathrm{mL} / \mathrm{m}^{2}\right)$, CI $\left(\mathrm{L} / \mathrm{min} / \mathrm{m}^{2}\right)$, systemic vascular resistance index (SVRI, dyne $\left.x \sec \times \mathrm{m}^{2} / \mathrm{cm}^{5}\right), \mathrm{dP} / \mathrm{dt}_{\max }$ ( $\mathrm{mmHg} / \mathrm{sec}$ ), and CCE (units). These hemodynamic data were acquired at 14 pre-defined time points: during transfer to the operation room (baseline measurement in the supine position $\mathrm{T} 0$ and lateral position $\mathrm{T} 1$ ), immediately following the application of spinal anesthesia (T2) and six further time points thereafter $(2,4,6,8,10$ and 12 min post-anesthesia while in the supine position-T3 to T8), during incision of the peritoneum (T9), during incision of the uterus (T10), during delivery (T11), immediately after the administration of oxytocin (T12), and at the completion of surgery (T13). Hypotension was defined as a decrease in SBP by up to $20 \%$ from baseline or a systolic blood pressure $<80 \mathrm{mmHg}$. Hypotension was treated with a bolus of $50 \mu \mathrm{g}$ of phenylephrine, or $15 \mathrm{mg}$ of ephedrine, depending on HR. If the HR exceeded 60 beats/min, we administered phenylephrine; if the HR below 60 beats/min, then we administered patients with ephedrine.

Immediately after the umbilical cord was clamped, we injected the uterine body of each patient with a rapid bolus of $10 \mathrm{U}$ of oxytocin diluted in $2 \mathrm{~mL}$ of saline. A separate infusion of $10 \mathrm{U}$ of oxytocin (in $50 \mathrm{~mL}$ of saline) was intravenous dropped over a period of $5 \mathrm{~min}$.

\section{Statistical analysis}

Unless otherwise stated, we have expressed descriptive statistics as means with standard deviations (SDs). Raw data was tested for normality using the KolmogorovSmirnov and Bartlett tests. Hemodynamic variables were compared between baseline and different timepoints after anesthesia using analysis of variance (ANOVA) for repeated measurements using SPSS version 23 (Chicago, IL, USA). 
Table 1 Demographic data

\begin{tabular}{lc}
\hline Patient characteristics & Means \pm SD $(\mathrm{n}=17)$ \\
\hline Age (years) & $32.08 \pm 4.46$ \\
Height $(\mathrm{cm})$ & $162.32 \pm 4.58$ \\
Body weight $(\mathrm{kg})$ & $74.37 \pm 9.80$ \\
BMl (at delivery, $\left.\mathrm{kg} / \mathrm{m}^{2}\right)$ & $28.26 \pm 2.89$ \\
Gestational age (weeks) & $39.17 \pm 0.67$ \\
HCT (\%) & $34.92 \pm 2.57$ \\
Incidence of hypotension, $\mathrm{n}(\%)$ & $4(23.53)$ \\
\hline
\end{tabular}

$\mathrm{SD}$, standard deviation; BMI, body mass index; HCT, hematocrit.

Table 2 Anesthesia detail

\begin{tabular}{lc}
\hline Anesthetic data & $\begin{array}{c}\text { Means } \pm \text { SD or median } \\
\text { [inter-quartile range] }(\mathrm{n}=17)\end{array}$ \\
\hline 0.5\% isobaric ropivacaine $(\mathrm{mL})$ & $2.4[2.4-2.4]$ \\
Level of anesthesia at incision, & $\mathrm{T} 6[\mathrm{~T} 4-\mathrm{T} 6]$ \\
dermatome & $81[72-93]$ \\
Anesthesia time (min) ${ }^{\mathrm{a}}$ & $4(23.53)$ \\
Vasopressors administration, $\mathrm{n}(\%)$ & $0.8[0.6-1.0]$ \\
Fluid administration (L) & $0.3[0.2-0.5]$ \\
Estimated blood loss (L) & $0.2[0.1-0.3]$ \\
Urine output (L) & $3(17.65)$ \\
Incidence of IONV, $\mathrm{n}(\%)$ &
\end{tabular}

Values are mean $\pm S D$, median [inter-quartile range] if not specified. " anesthesia time is from the time of anesthesia induction to the time of departure from operating room after conclusion of surgery. SD, standard deviation; IONV, intraoperative nausea and vomiting.

If ANOVA revealed interactions that were significant, then the data were analyzed further using the Bonferroni post hoc multiple comparisons test. This test allowed us to determine whether the pre-defined time points yielded data that were significantly different from baseline values and previous timepoints. Differences were considered to be statistically significant if $\mathrm{P}<0.05$.

\section{Results}

\section{Demographics}

We recruited 20 patients into this study. We excluded three patients due to inappropriate arterial pressure signal acquisition, and a further two patients due to the inadequate blockade of spinal anesthesia. Thus, 17 patients were included in our final analyses. The demographics of our patient characteristics, and specific anesthetic details are given in Tables 1,2.

\section{HR and blood pressure}

Table 3 and Figure 1 depict mean values for the hemodynamic data at pre-defined timepoints. There were no statistically significant alterations in HR throughout the procedure (Figure 1A). However, HR gradually increased after spinal anesthesia and reached a peak at T8 (12 min after spinal anesthesia). Following incision of the peritoneum, the HR returned to almost baseline levels and increased immediately after the administration of oxytocin (T12).

Following spinal anesthesia, there was a significant reduction in MAP at T8 and T12 (12 min after spinal anesthesia and the administration of oxytocin) $(\mathrm{P}<0.001)$. This reduction in MAP was reversely correlated with HR (Figure 1B).

The Pdic arises as a direct result of a short period of backwards blood flow that occurs immediately prior to the aortic valve closing. The Pdic is also related to the MAP and mean peripheral vascular resistance. In this study, Pdic was significantly reduced at $\mathrm{T} 7(\mathrm{P}=0.0013)$, T8 $(\mathrm{P}<0.001)$, and $\mathrm{T} 12(\mathrm{P}<0.001)(10$ to $12 \mathrm{~min}$ after spinal anesthesia, and following the administration of oxytocin) (Figure 1C).

\section{CI and SVR}

We observed a significant reduction in CI immediately following spinal anesthesia at the $\mathrm{T} 7(\mathrm{P}=0.0035)$ and $\mathrm{T} 8$ $(\mathrm{P}<0.001)$ time points. The mean maximum reduction in CI was $33.03 \%$ from baseline, which occurred at T8 time point $(\mathrm{P}<0.001)$ (Figure $1 D)$. After incision of the peritoneum, there was a significant increase in $\mathrm{CI}$ compared to T8 $(\mathrm{P}<0.001)$. Furthermore, $\mathrm{CI}$ was significantly reduced immediately after the administration of oxytocin $(\mathrm{P}=0.021$ compared to $\mathrm{T} 0, \mathrm{P}<0.001$ compared to T11). There was a reverse correlation between CI and SVRI. We observed a significant increase in SVRI between baseline and after spinal anesthesia. SVRI increased progressively at T7 and T8 and reached a peak at T8 that was $31.37 \%$ higher than baseline $(\mathrm{P}<0.001)$. Thereafter, SVRI showed a significant reduction after incision of the peritoneum compared to $\mathrm{T} 8$ $(\mathrm{P}<0.001)$ (Figure 1E). 


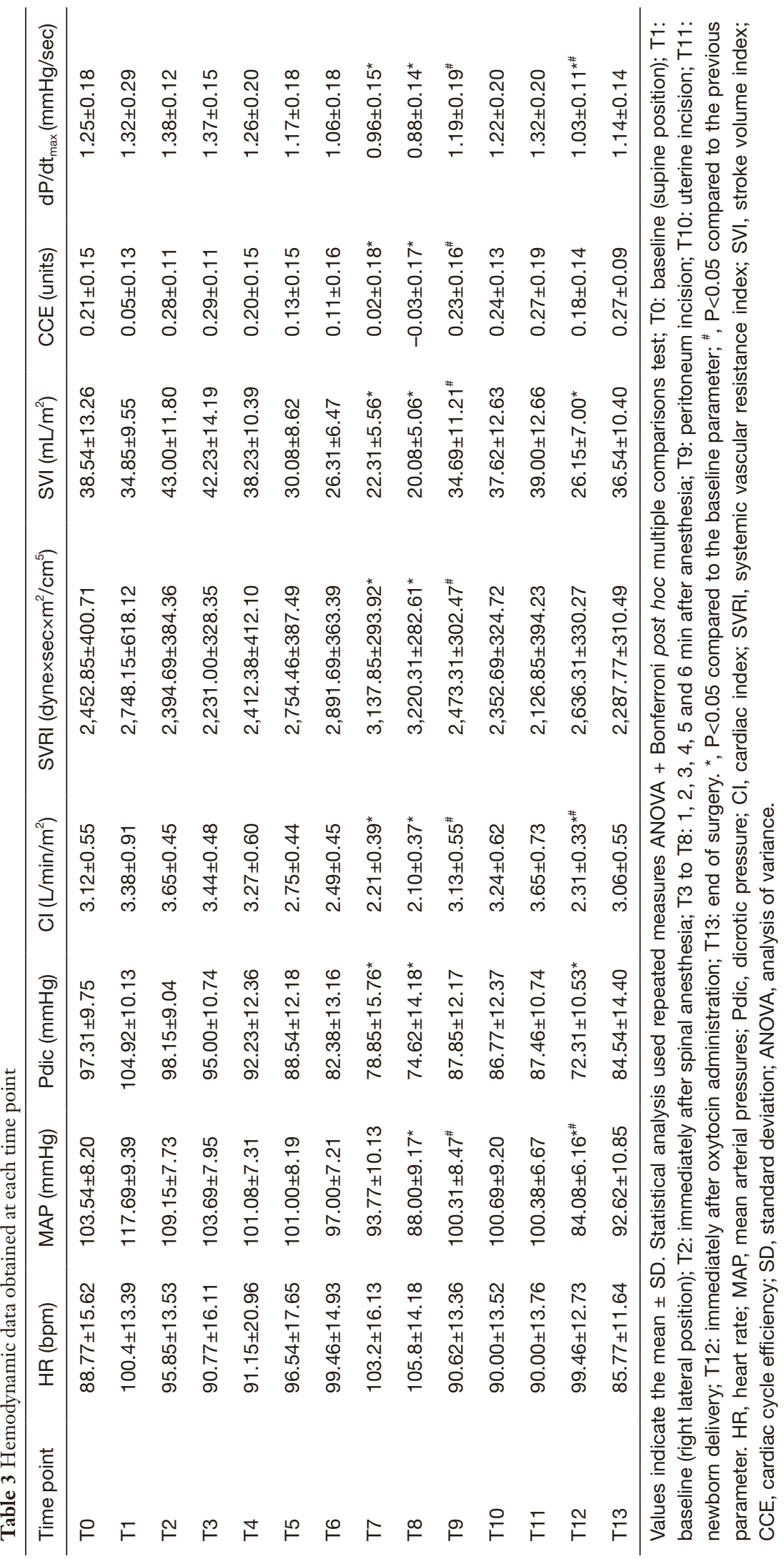


A

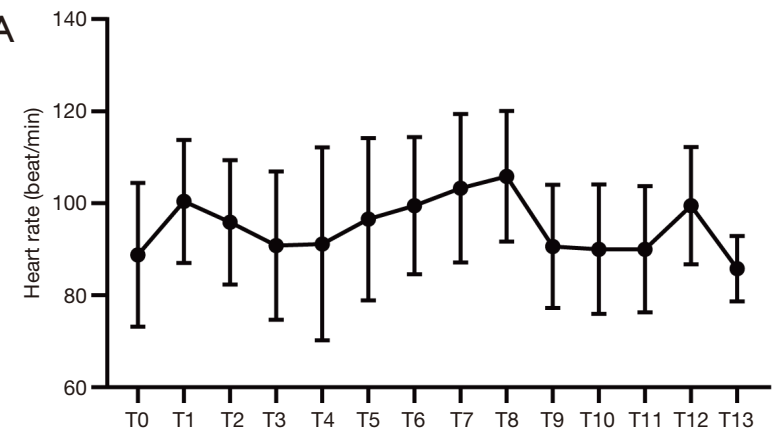

C

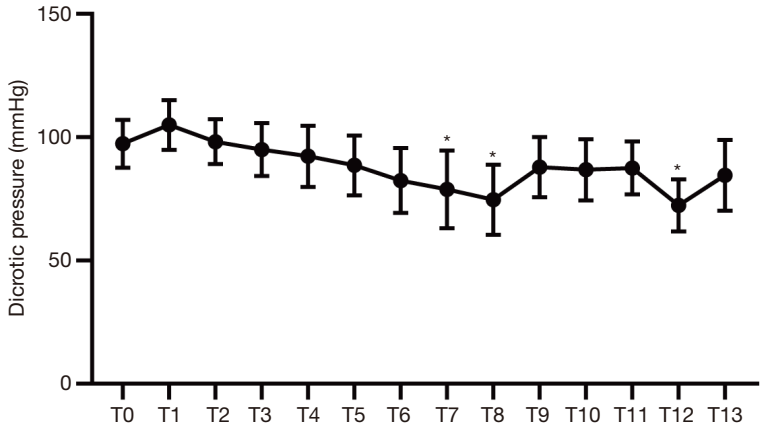

$\mathrm{E}$

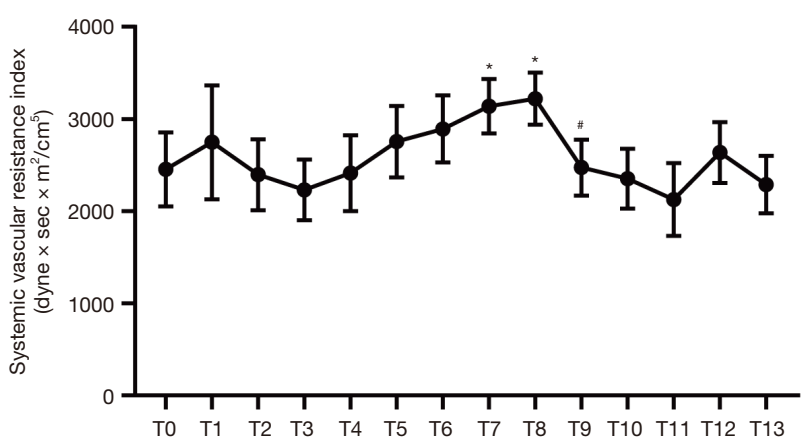

G

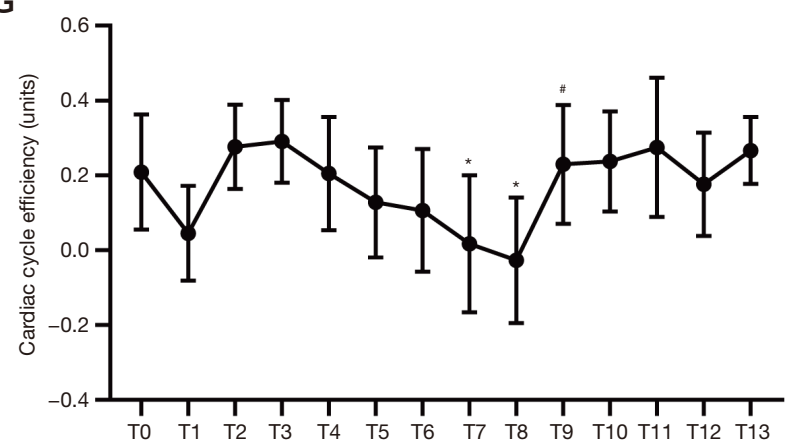

B

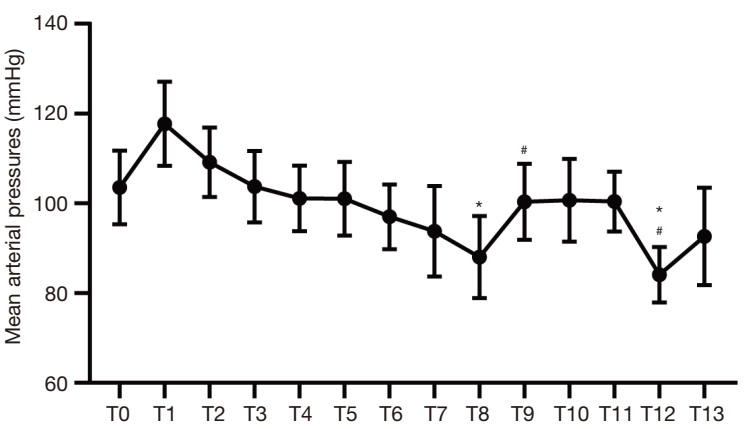

D

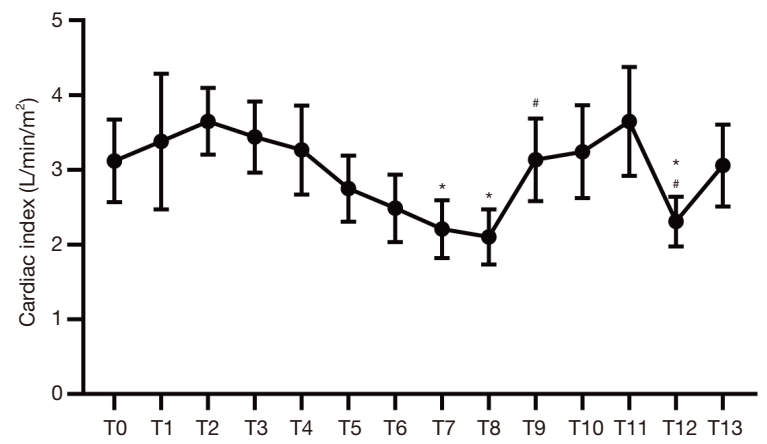

$\mathrm{F}$

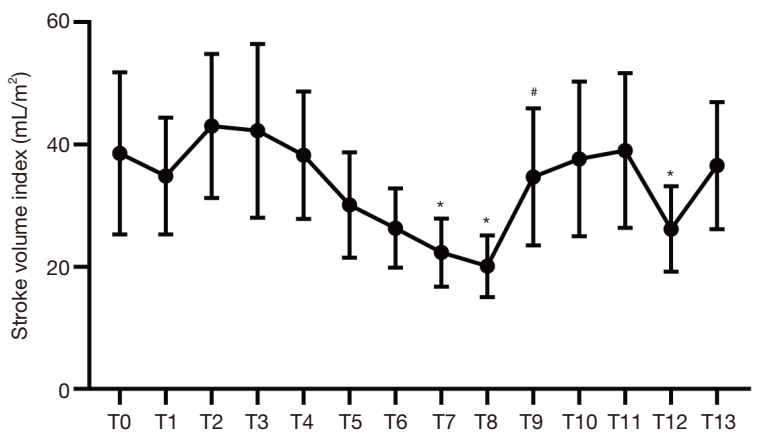

$\mathrm{H}$

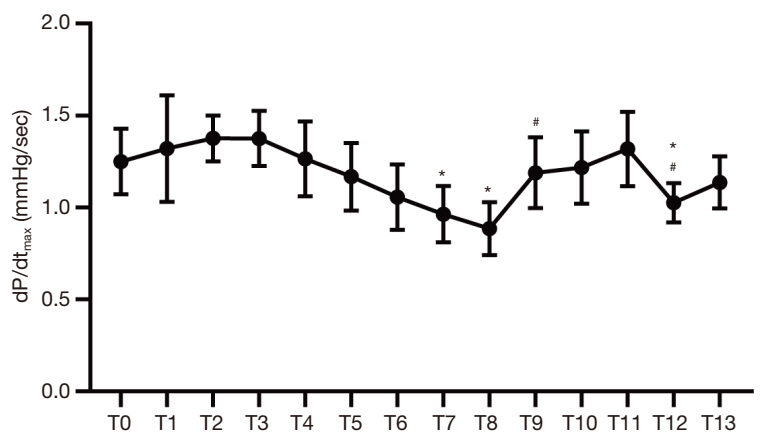

Figure 1 Hemodynamic parameters change at pre-defined time points during cesarean delivery under spinal anesthesia. (A) HR; (B) MAP; (C) Pdic; (D) CI; (E) SVRI; (F) SVI; (G) CCE; (H) dP/dt $t_{\max }$. Data were presented as mean \pm SD. *, P<0.05 compared to the baseline values; \#, $\mathrm{P}<0.05$ compared to the previous values. HR, heart rate; MAP, mean arterial pressure; Pdic, dicrotic pressure; CI, cardiac index; SVRI, systemic vascular resistance index; SVI, stroke volume index; CCE, cardiac cycle efficiency; SD, standard deviation. 


\section{SVI, CCE, and $d P / d t_{\max }$}

We also observed a significant reduction in SVI, CCE, and $\mathrm{dP} / \mathrm{dt}_{\max }$, following the induction of spinal anesthesia at $\mathrm{T} 7$ and T8 $(\mathrm{P}<0.05$ for all). After incision of the peritoneum (T9), all of these three parameters increased significantly compared to $\mathrm{T} 8(\mathrm{P}<0.05$ for all) (Figure 1F,G,H). Following the administration of oxytocin (T12), there was a significant reduction in SVI and $\mathrm{dP} / \mathrm{dt}_{\max }$ compared to the baseline level $(\mathrm{P}<0.05$ for all).

\section{Discussion}

The research presented herein describes the profiles of various maternal hemodynamic data under spinal anesthesia during cesarean delivery. We acquired these data by applying PRAM of MOSTCARE system in 32 healthy pregnant women. We observed significant reductions in MAP, Pdic, CI, SVI, CCE, and $\mathrm{dP} / \mathrm{dt}_{\max }$, after spinal anesthesia. During the same time period, there was a significant increase in SVRI. After incision of the peritoneum, all of these hemodynamic parameters returned to levels similar to those seen at baseline. Furthermore, there was also a significant reduction in MAP, Pdic, and CI after the administration of oxytocin. Consequently, the application of this minimally invasive system for monitoring hemodynamic data may improve the management of hemodynamics during obstetric anesthesia.

Previous research has indicated that hypotension induced by spinal anesthesia commonly occurs after sensory blockade at levels above $\mathrm{T} 5$ or $\mathrm{T} 4$ in pregnant patients $(10,11)$. These findings concur with the fact that nerve fibers that control the vasomotor tone of the arterial and venous vessels originate from the T5-L1 levels and that cardioaccelerator fibers originate from the T1-T4 levels (12). The high incidence of hypotension following spinal anesthesia in pregnant women is also associated with the rapidly ascending level of sensory blockade (13). The slow ascension rate of the sensory block level results in a low incidence of hypotension; this is because of the fact that a slower-onset sympathetic block allows more time for physiological compensation. Furthermore, body position, and the density of anesthetics delivered locally, are considered to represent important factors that are related to spinal anesthesia-induced hypotension (14). In this study, pregnant women received spinal-epidural anesthesia whilst lying in the right-lateral position. These patients were administered with $12 \mathrm{mg} 0.5 \%$ isobaric ropivacaine and the sensory block level was controlled at the T6 level just 10 min after the anesthetic was administered. We observed that MAP gradually decreased as the sensory block level ascended and that there was a significant reduction $12 \mathrm{~min}$ after spinal anesthesia (at T8 time point). The incidence of spinal anesthesia-induced hypotension observed in this study was $21.88 \%$. This result is consistent with previous studies that controlled sensory block level and ascension rate while administering isobaric ropivacaine in the rightlateral position $(13,14)$.

CO and SVR changes after spinal anesthesia are still controversial. Previous studies have reported that CO increases significantly immediately after spinal anesthesia, followed by a significant reduction in $\mathrm{CO}$ subsequent to the administration of vasoconstriction drugs $(15,16)$. A rapid and profound reduction in SVR after spinal anesthesia often precedes the reduction in MAP (17). Our data show an upwards trend in CI immediately after spinal anesthesia. Thereafter, CI gradually declined and was significantly reduced at 10 and $12 \mathrm{~min}$ after the administration of anesthetics. However, there was a reverse correlation between CI and SVRI. SVRI show a downwards trend immediately after spinal anesthesia and increased progressively 10 minutes later. After incision of the peritoneum, these two hemodynamic parameters returned to levels similar to those at baseline. The differences between our findings and those from previous studies may be due to differences in the sensory block level, and perhaps the administration of vasoconstrictors. In this study, the sensory block level was controlled at the T6 level. As a result, there was little impact on vasomotor tone $(10,11)$. We believe that the upwards trend shown by CI following spinal anesthesia was due to the reduction in SVRI; this was caused mainly by vasodilation and a compensatory increase in HR. The significant reduction of CI at 10 and 12 min after the administration of anesthetics is mainly due to compression of the maternal abdominal aorta and the inferior vena cava (18). We also observed compensatory changes in SVRI that were considered to be correlated with changes in $\mathrm{CO}$. When compression of the aorta and inferior vena cava was relieved by incision of the peritoneum, these two hemodynamic parameters immediately returned to baseline levels.

The major factors influencing SVI are preload, afterload, and contractility (19). In the present study, we observed a significant reduction of SVI following spinal anesthesia. Two reasons may explain these changes in SVI. First, compression of the inferior vena cava produces a reduced 
return of blood to the heart, thus resulting in a reduction in preload (18). Second, the reduction in SVI was probably the result of physiological compensation caused by an increase in HR. Previous studies demonstrated that arterial $\mathrm{dP} / \mathrm{dt}_{\max }$ can accurately reflect left ventricular contractility and preload $(20,21)$. In this study, we found that $\mathrm{dP} / \mathrm{dt}_{\max }$ was significantly lower than baseline after spinal anesthesia at $\mathrm{T} 7$ and $\mathrm{T} 8$ time points. This result indicates that compression of the inferior vena cava and subarachnoid block have an impact on left ventricular contractility and preload. Following spinal anesthesia, the gradually increasing compression of the inferior vena cava may lead to myocardial dysfunction and the loss of preload. Similarly, incision of the peritoneum can relieve compression on the vena cava and the fluctuations in these two hemodynamic parameters.

In addition to allowing the investigation of $\mathrm{CO}$, the PRAM system also has the capability to provide us with a new parameter for continuous hemodynamic monitoring, i.e., CCE. The CCE describes a ratio that incorporates hemodynamic work performed/energetic expenditure and provides an indicator of cardiac hemodynamic performance $(22,23)$. A reduction in CCE therefore reflects an increase in the amount of energy used by the cardiovascular system to maintain the same balance in hemodynamics (24). In this study, we observed a significant reduction in CCE after spinal anesthesia. This means that the cardiovascular system consumed more energy to maintain hemodynamic balance. We believe that this mechanism involves a range of factors and is mainly caused by sympathetic blockade and compression of the inferior vena cava.

Finally, our analyses demonstrated significant fluctuations in hemodynamic parameters following the administration of oxytocin. The hemodynamic effects accompanied by an infusion or bolus of oxytocin are welldocumented (25). Oxytocin receptors are widely expressed in the cardiovascular system. Activation of oxytocin receptors in cardiac tissue induces the release of atrial natriuretic peptide; upon binding to endothelial receptors, this peptide stimulates the release of nitric oxide (26). These events ultimately lead to vasodilation and a reduction in HR $(15,27)$. In this study, the CI and SVI were significantly reduced immediately after the administration of oxytocin; this was accompanied by a slight increase in HR and SVRI. We suppose that this hemodynamic response is related to the high-dose oxytocin regimen that we applied in the current study $(10 \mathrm{U}$ oxytocin administered as a rapid bolus and $10 \mathrm{U}$ oxytocin rapid intravenous drip). The precise hemodynamic effects of a high-dose oxytocin regimen need to be investigated further.

The main limitation of this study is that the reliability of PRAM is dependent upon the fact that the arterial pressure signal is of good quality (28). Patients with serious hypotension may cause inappropriate signal acquisition. As a result, the MostCare monitor might provide incorrect values in the event that the pressure signal is not stable. Therefore, we did not perform measurements in pregnant women with severe post-spinal hypotension. Further research should focus on the hemodynamic profiles of patients with serious post-spinal hypotension.

In summary, for the first time, we report the use of a minimally invasive monitor based on PRAM to evaluate changes in the hemodynamics of pregnant women undergoing spinal anesthesia for cesarean delivery. Using this system, we observed several key fluctuations in the hemodynamic status of patients undergoing spinal anesthesia that were associated with the anesthetic technique, compression of the aorta and inferior vena cava, and the administration of oxytocin. Gaining a better understanding of how hemodynamics can fluctuate during obstetric procedures involving spinal anesthesia may provide us with new strategies to reduce both maternal and neonatal morbidity. We propose that comprehensive continuous hemodynamic monitoring may help us improve the management of anesthetics in obstetric procedures and allow us to provide individualized management plans for obstetrics patients.

\section{Acknowledgments}

The authors would like to express their gratitude to EditSprings (https://www.editsprings.com/) for the expert linguistic services provided.

Funding: This study was supported by Shanghai Municipal Commission of Health and Family Planning, China (No. 20164Y0263).

\section{Footnote}

Reporting Checklist: The authors have completed the MDAR reporting checklist. Available at http://dx.doi.org/10.21037/ apm-21-598

Data Sharing Statement: Available at http://dx.doi. 
org/10.21037/apm-21-598

Peer Review File: Available at http://dx.doi.org/10.21037/ apm-21-598

Conflicts of Interest: All authors have completed the ICMJE uniform disclosure form (available at http://dx.doi. org/10.21037/apm-21-598). The authors have no conflicts of interest to declare.

Ethical Statement: The authors are accountable for all aspects of the work in ensuring that questions related to the accuracy or integrity of any part of the work are appropriately investigated and resolved. This observational study was approved by the Ethics Committee of International Peace Maternity \& Child Health Hospital, Shanghai Jiao Tong University, School of Medicine (GKLW2015-65). The study was conducted in accordance with the Declaration of Helsinki (as revised in 2013). All of the patients involved in our study provided written and informed consent.

Open Access Statement: This is an Open Access article distributed in accordance with the Creative Commons Attribution-NonCommercial-NoDerivs 4.0 International License (CC BY-NC-ND 4.0), which permits the noncommercial replication and distribution of the article with the strict proviso that no changes or edits are made and the original work is properly cited (including links to both the formal publication through the relevant DOI and the license). See: https://creativecommons.org/licenses/by-nc-nd/4.0/.

\section{References}

1. Hehir MP, Ananth CV, Siddiq Z, et al. Cesarean delivery in the United States 2005 through 2014: a populationbased analysis using the Robson 10-Group Classification System. Am J Obstet Gynecol 2018;219:105.e1-e11.

2. Heesen M, Klimek M, Hoeks SE, et al. Prevention of spinal anesthesia-induced hypotension during cesarean delivery by 5 -hydroxytryptamine- 3 receptor antagonists: a systematic review and meta-analysis and meta-regression. Anesth Analg 2016;123:977-88.

3. Ngan Kee WD. A random-allocation graded doseresponse study of norepinephrine and phenylephrine for treating hypotension during spinal anesthesia for cesarean delivery. Anesthesiology 2017;127:934-41.

4. Kranke P, Annecke T, Bremerich DH, et al. Anesthesia in obstetrics: Tried and trusted methods, current standards and new challenges. Anaesthesist 2016;65:3-21.

5. Langesæter E, Gibbs M, Dyer RA. The role of cardiac output monitoring in obstetric anesthesia. Curr Opin Anaesthesiol 2015;28:247-53.

6. Romagnoli S, Franchi F, Ricci Z, et al. The pressure recording analytical method (PRAM): technical concepts and literature review. J Cardiothorac Vasc Anesth 2017;31:1460-70.

7. Donati A, Carsetti A, Tondi S, et al. Thermodilution vs pressure recording analytical method in hemodynamic stabilized patients. J Crit Care 2014;29:260-4.

8. Alonso-Iñigo JM, Escribá FJ, Carrasco JI, et al. Measuring cardiac output in children undergoing cardiac catheterization: comparison between the Fick method and PRAM (pressure recording analytical method). Paediatr Anaesth 2016;26:1097-105.

9. Barthélémy R, Neuschwander A, Dramé F, et al. Monitoring haemodynamic response to fluid-challenge in ICU: comparison of pressure recording analytical method and oesophageal Doppler: a prospective observational study. Eur J Anaesthesiol 2019;36:135-43.

10. Chang JE, Kim H, Ryu JH, et al. Relationship between central obesity and spread of spinal anesthesia in female patients. Anesth Analg 2017;124:1670-3.

11. Fakherpour A, Ghaem H, Fattahi Z, et al. Maternal and anaesthesia-related risk factors and incidence of spinal anaesthesia-induced hypotension in elective caesarean section: a multinomial logistic regression. Indian J Anaesth 2018;62:36-46.

12. Ozkan Seyhan T, Orhan-Sungur M, Basaran B, et al. The effect of intra-abdominal pressure on sensory block level of single-shot spinal anesthesia for cesarean section: an observational study. Int J Obstet Anesth 2015;24:35-40.

13. Zhang N, He L, Ni JX. Level of sensory block after spinal anesthesia as a predictor of hypotension in parturient. Medicine (Baltimore) 2017;96:e7184.

14. Xu Z, Shen F, Zhang Y, et al. Combined spinal-epidural anesthesia with hypobaric ropivacaine in sitting position significantly increases the incidence of hypotension in parturients undergoing cesarean section. J Obstet Gynaecol Res 2017;43:669-75.

15. Dyer RA, Reed AR, van Dyk D, et al. Hemodynamic effects of ephedrine, phenylephrine, and the coadministration of phenylephrine with oxytocin during spinal anesthesia for elective cesarean delivery. Anesthesiology 2009;111:753-65.

16. Orbach-Zinger S, Bizman I, Firman S, et al. Perioperative 
noninvasive cardiac output monitoring in parturients undergoing cesarean delivery with spinal anesthesia and prophylactic phenylephrine drip: a prospective observational cohort study. J Matern Fetal Neonatal Med 2019;32:3153-9.

17. Liu Y, Pian-Smith MC, Leffert LR, et al. Continuous measurement of cardiac output with the electrical velocimetry method in patients under spinal anesthesia for cesarean delivery. J Clin Monit Comput 2015;29:627-34.

18. Lee SW, Khaw KS, Ngan Kee WD, et al. Haemodynamic effects from aortocaval compression at different angles of lateral tilt in non-labouring term pregnant women. Br J Anaesth 2012;109:950-6.

19. Pingitore A, Aquaro GD, Lorenzoni V, et al. Influence of preload and afterload on stroke volume response to lowdose dobutamine stress in patients with non-ischemic heart failure: a cardiac MR study. Int J Cardiol 2013;166:475-81.

20. Blaudszun G, Licker MJ, Morel DR. Preload-adjusted left ventricular $\mathrm{dP} / \mathrm{dtmax}$ : a sensitive, continuous, load-independent contractility index. Exp Physiol 2013;98:1446-56.

21. Morimont P, Lambermont B, Desaive T, et al. Arterial $\mathrm{dP} / \mathrm{dtmax}$ accurately reflects left ventricular contractility during shock when adequate vascular filling is achieved. BMC Cardiovasc Disord 2012;12:13.

Cite this article as: Zhang $\mathrm{X}, \mathrm{Xu} \mathrm{T}$, Jia $\mathrm{L}, \mathrm{Cao} \mathrm{H}, \mathrm{Xu} \mathrm{Z}$. Continuous cardiovascular hemodynamics monitoring with pressure recording analytical method in patients under spinal anesthesia for elective cesarean section: a pilot study. Ann Palliat Med 2021;10(7):7184-7193. doi: 10.21037/apm-21-598
22. Romano SM. Cardiac cycle efficiency: a new parameter able to fully evaluate the dynamic interplay of the cardiovascular system. Int J Cardiol 2012;155:326-7.

23. Scolletta S, Ranaldi G, Carlucci F, et al. Relationship between $\mathrm{N}$-terminal pro-B-type natriuretic peptide (NtproBNP) and cardiac cycle efficiency in cardiac surgery. Biomed Pharmacother 2010;64:511-5.

24. Messina A, Romano SM, Bonicolini E, et al. Cardiac cycle efficiency and dicrotic pressure variations: new parameters for fluid therapy: an observational study. Eur J Anaesthesiol 2017;34:755-63.

25. Gutkowska J, Jankowski M, Antunes-Rodrigues J. The role of oxytocin in cardiovascular regulation. Braz J Med Biol Res 2014;47:206-14.

26. Japundžić-Žigon N. Vasopressin and oxytocin in control of the cardiovascular system. Curr Neuropharmacol 2013;11:218-30.

27. Rosseland LA, Hauge TH, Grindheim G, et al. Changes in blood pressure and cardiac output during cesarean delivery: the effects of oxytocin and carbetocin compared with placebo. Anesthesiology 2013;119:541-51.

28. Scolletta S, Romano SM, Biagioli B, et al. Pressure recording analytical method (PRAM) for measurement of cardiac output during various haemodynamic states. Br J Anaesth 2005;95:159-65. 